ReCEIVEd 2007 August 6; ACCEPTEd 2007 SePtember 23

Preprint typeset using LTEX style emulateapj v. 08/22/09

\title{
DISCOVERY OF COHERENT MILLISECOND X-RAY PULSATIONS IN AQL X-1
}

\author{
P. Casella ${ }^{1}$, D. Altamirano ${ }^{1}$, A. Patruno ${ }^{1}$, R. Wijnands ${ }^{1}$, And M. VAn Der Klis ${ }^{1}$ \\ Received 2007 August 6; accepted 2007 September 23
}

\begin{abstract}
We report the discovery of an episode of coherent millisecond X-ray pulsation in the neutron star low-mass $\mathrm{X}$-ray binary Aql X-1. The episode lasts for slightly more than 150 seconds, during which the pulse frequency is consistent with being constant. No X-ray burst or other evidence of thermonuclear burning activity is seen in correspondence with the pulsation, which can thus be identified as occurring in the persistent emission. The pulsation frequency is $550.27 \mathrm{~Hz}$, very close $(0.5 \mathrm{~Hz}$ higher) to the maximum reported frequency from burst oscillations in this source. Hence we identify this frequency with the neutron star spin frequency. The pulsed fraction is strongly energy dependent, ranging from $<1 \%$ at $3-5 \mathrm{keV}$ to $>10 \%$ at $16-30 \mathrm{keV}$. We discuss possible physical interpretations and their consequences for our understanding of the lack of pulsation in most neutron star low-mass X-ray binaries. If interpreted as accretion-powered pulsation, Aql X-1 might play a key role in understanding the differences between pulsating and non-pulsating sources.
\end{abstract}

Subject headings: pulsars: individual (Aql X-1) - stars: neutron - X-rays: binaries

\section{INTRODUCTION}

Accretion-powered millisecond X-ray pulsars (hereinafter AMSPs) had been predicted in the early 1980s as the progenitors of millisecond radio pulsars (Backer et al. 1982; Alpar et al. 1982). The first observational indication that neutron stars in low-mass X-ray binaries (LMXBs) rotate rapidly came in 1996 with the discovery of slightly drifting in frequency millisecond oscillations during thermonuclear X-ray bursts (for a review see Strohmaver \& Bildsten 2006). However it was not until 1998 that the first AMSP was discovered (Wijnands \& van der Klis 1998). Since then, a total of eight AMSPs have been found out of the $>150$ LMXBs known up to date (Liu et al. 2007).

Since the theoretical prediction of the existence of AMSPs was made, the main issue remained to explain the lack of pulsation in the persistent X-ray emission of the majority of LMXBs. In recent decades many theoretical efforts have been made to explain this, the main question remaining whether the pulsation is hidden from the observer or not produced at all. At present, the scenarios most often considered are: (a) the magnetic field in non-pulsating LMXBs is too weak to allow channeling of the matter onto the magnetic poles; (b) the magnetic field has comparable strength inside most LMXB neutron stars, but in the large majority of them it has been "buried" by accretion, resulting in a very low surface magnetic field (e.g., Cumming et al. 2001) which again is too weak to allow channeling of matter. After the accretion stops, the magnetic field eventually emerges from the neutron star surface and assumes its intrinsic value; (c) pulsations are produced in all LMXBs, but in most of them they are attenuated by a surrounding scattering medium that washes out the coherent beamed pulsation (Brainerd \& Lamb 1987; Kylafis \& Klimis 1987; Titarchuk et al. 2003); (d) the pulsations are attenuated by gravitational lensing from the neutron star (e.g., Meszaros et al. 1988).

From an observational point of view, since the discovery of the first AMSP efforts have been focused on finding differ-

\footnotetext{
${ }^{1}$ Astronomical Institute, "Anton Pannekoek", University of Amsterdam, Kruislaan 403, 1098 SJ Amsterdam, The Netherlands. E-mail: casella@science.uva.nl
}

ences between the sources showing pulsation and those that do not, in order to test different theoretical hypotheses. Possible observed differences so far are the orbital period (which is on average shorter in AMSPs than in other LMXBs, see e.g., Kaaret et al. 2006) and the time-averaged accretion rate (which is considered to be on average smaller in AMSPs than in other LMXBs, see e.g., Galloway 2006). However, although it is probable that orbital period and time-averaged accretion rate play an important role in the determination of AMSP properties, the reason for the lack of pulsation in most of LMXBs still has to be found.

The properties of the seventh discovered AMSP (HETE J1900.1-2455, Kaaret et al. 2006; Galloway et al. 2007) gave new insights to this issue. This source has a much higher inferred time-averaged accretion rate than in the other AMSPs. The pulsation became undetectable after two months from the beginning of the outburst, at strong variance with the other AMSPs in which pulsations were always detectable until the end of the outbursts. This has been interpreted as evidence for burying of the magnetic field by the accreted material during the outburst (Galloway et al. 2007).

Transient highly coherent pulsations were also observed in 4U 1636-53 (Strohmayer \& Markwardt 2002) during an $\sim 800 \mathrm{~s}$ interval. However, the pulsations were detected at the flux maximum of a superburst, hence they were likely nuclearpowered. This is different compared to the seven AMSP, where pulsations were interpreted in terms of an hotspot resulting from magnetic channeling of matter onto the neutron star surface.

We are searching the full Rossi X-ray Timing Explorer (RXTE) archive data for coherent pulsations (see also Altamirano et al. 2007). In this letter we report the discovery of an episode of coherent millisecond X-ray pulsarions in the neutron star binary Aq1 X-1. The LMXB recurrent X-ray transient Aql X-1 is an atoll source (Reig et al. 2000) wich shows $\mathrm{kHz}$ quasi-periodic oscillations, X-ray bursts and burst oscillations (Zhang et al. 1998b). An orbital period of $18.95 \mathrm{hr}$ was obtained by optical measurments (Chevalier \& Ilovaisky 1991; Welsh et al.2000). The presumed onset of the propeller effect (Illarionov \& Sunyaev 1975; Stella et al. 1986) allowed Campana et al. (1998) to estimate a value for the magnetic 


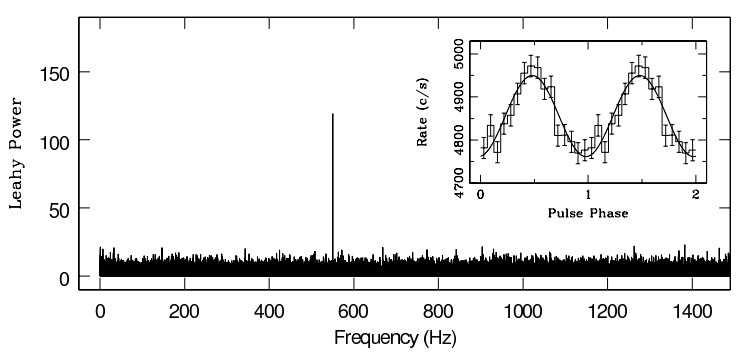

FIG. 1.- Power spectrum of the last $128 \mathrm{~s}$ data interval of observation 30188-03-05-00 (see Fig. 2). The power spectrum was obtained from the full bandpass event mode data. The frequency resolution is $1 / 128 \mathrm{~Hz}$. The pulse is clearly visible at $\sim 550 \mathrm{~Hz}$. In the inset we plot the $2-60 \mathrm{keV}$ light curve folded at the pulsation period. Two cycles are plotted for clarity. The solid line shows the best sinusoidal fit.

field of $1-3 \times 10^{8}\left(\frac{D}{2.5 \mathrm{kpc}}\right) \mathrm{G}$, consistent with the magnetic field expected (and later on measured) in AMSPs (see also Zhang et al. 1998a).

We analyzed RXTE archive data of Aql X-1 and found that the source showed coherent pulsation at a frequency near the neutron star spin frequency (as inferred from burst oscillations) in its persistent emission for 150 seconds during the peak of its 1998 outburst. The discovery of this pulsation episode may provide new insights on the issue of why some LMXBs pulse and some do not, after many years of debate.

\section{DATA ANALYSIS}

We analyzed the whole PCA public archive of Aql X-1, searching for coherent pulsation in its persistent emission. A total of 363 observations were analysed. In addition to the two Standard Modes always present, the PCA data in different observations were obtained in two different high-time resolution modes (either Event Mode $(125 \mu \mathrm{s})$ or GoodXenon $(\sim 1 \mu \mathrm{s})$ ); to all data we applied barycenter correction for Earth and satellite motion. For each observation we computed fast Fourier transforms from 128s data intervals (after filtering for the presence of X-ray bursts) and obtained power spectra with a Nyquist frequency of $4096 \mathrm{~Hz}$ and a frequency resolution of $1 / 128 \mathrm{~Hz}$. We searched all power spectra for significant power at frequencies close to the expected pulse frequency $(\sim 550$ $\mathrm{Hz}$ ). Only one detection was found with a Leahy power of 120 (Leahy et al. 1983), corresponding to a single trial chance probability of $9 \times 10^{-27}$ at a frequency of $550.27 \mathrm{~Hz}$ (see Fig. 11 .

The single trial significance of the pulsation is $11 \sigma$. Once we take into account the number of trials (i.e. the number of frequency bins times the number of $128 \mathrm{~s}$ power spectra we searched) the significance becomes $9 \sigma\left(3 \times 10^{-17}\right.$ chance probability). Combined with its frequency being near that of the previously observed burst oscillations, we conclude that the pulsation is real and intrinsic to $\mathrm{Aql} \mathrm{X}-1$ and represents the spin of the neutron star in this source. A full description of the total data analysis is beyond the scope of this letter and will be the subject of a forthcoming publication. Here we focus on the detailed analysis performed of the single observation where pulsation was discovered.

The pulsation was detected at the end of a 1600-second long observation starting on 1998 March 10 at 22:28 UT (ObsId: 30188-03-05-00). All five proportional counter units were active during the whole observation, with a total $2-60 \mathrm{keV}$ count rate varying between 4500 and 5500 counts/sec. During this observation high-time resolution data were available in Event Mode with $125 \mu$ s time resolution and 64 energy channels over the 2-60 keV instrument bandpass. The light curve of the ob-

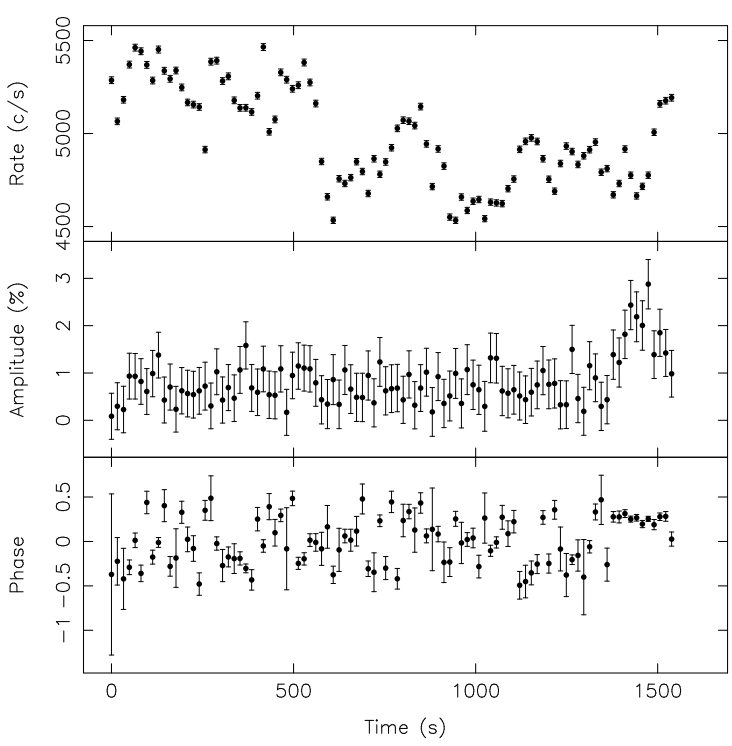

FIG. 2.- Top panel: 2-60 light curve with 16 seconds bin size. Bottom panels: Amplitude (middle) and phase (bottom) by epoch folding technique every 16 seconds, vs. time. Values of amplitude and phase between 0 and $\sim 1400 \mathrm{~s}$ are consistent with those expected from Poissonian noise.

servation is shown in the top panel of Figure 2

The high power was detected in the last $128 \mathrm{~s}$ interval of the observation. By using an epoch folding technique we obtain a period estimate of $1.8172746 \mathrm{~ms}$. The folded profile was well modeled with a sinusoidal component with $1.9 \pm 0.2 \%$ amplitude (see inset of Fig. 11). No second harmonic could be detected, with a $95 \%$ upper limit on the amplitude of $0.8 \%$. To study the evolution and the properties of the signal we extracted a light curve with maximum time resolution, divided it into 16-second segments and determined the pulsation amplitude and phase in each segment by an epoch folding technique (see Fig. 2). The pulsation is detected for $\sim 150$ seconds at the end of the observation; its amplitude gradually increases and then decreases again over these 150 seconds. To estimate the accuracy of our period measurement we performed a linear fit to the phases in the interval where pulsations are detected. Over this interval the phase is consistent with being constant. From a $3 \sigma$ upper limit of $10^{-3} \mathrm{~s}^{-1}$ on the slope, we derive a best period measurement of $1.817275(3) \mathrm{ms}$. The short duration of the pulsation did not allow us to obtain any useful upper limit on the frequency drift. From the number of cycles we were able to phase connect, we can estimate the coherence of the pulsation as $\mathrm{Q} \geq 8 \times 10^{4}$. No correction for the binary orbital motion was possible, given the small expected drift ( $<2 \mathrm{mHz}$ in 150 seconds) and the large uncertainity on the orbital phase ( $\sim 80 \%$, applying the orbital solution from Welsh et al. 2000).

In order to study the energy dependence of the pulsation we extracted light curves with maximum time resolution in different energy ranges and determined the pulsation amplitude and phase in each of them. In Figure 3, we show the amplitude vs. energy diagram. The pulsations have a strong energy dependence, being not detected at low energies (with an upper limit on its amplitude of $\sim 1 \%$ below $5 \mathrm{keV}$ ) and strong at high energies.

\section{DISCUSSION}

We discovered millisecond X-ray pulsations in the persistent X-ray emission of the LMXB Aql X-1. The pulsation is transient, present for only $\sim 150$ seconds and has an aver- 


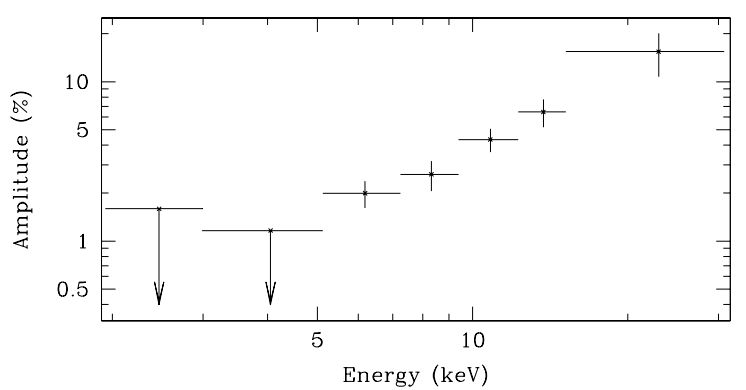

FIG. 3.- Amplitude of the pulsation as a function of energy. $1 \sigma$ errors are shown.

age fractional amplitude (over the full 2-60 keV instrumental bandpass) of $\sim 2 \%$, increasing up to $>10 \%$ at energies above $\sim 16 \mathrm{keV}$. It appears and disappears gradually, on a time scale of a few tens of seconds, with a $3 \sigma$ upper limit on its amplitude of $0.7 \%$ during the $\sim 128$ seconds before its appearance.

This pulsating episode appears to be unique in the whole RXTE archive of Aql X-1 observations. In one third of the observations (corresponding to an exposure of $500 \mathrm{ks}$ ) the count rate is high enough to allow the detection (3- $\sigma$, single trial) of a similar pulsating episode as the one we observed. Even considering only these data (instead of the total analysed exposure of $1300 \mathrm{ks}$ ) we obtain a recurrence rate smaller than $3 \times 10^{-4}$. Such extreme rarety in itself is extremely informative. Any physical interpretation must not only in fact explain the appearance of the pulsation, but also its extremely low occurrence rate.

The frequency of the pulsation is $550.27 \mathrm{~Hz}, \sim 0.53 \mathrm{~Hz}$ higher than the reported asymptotic frequencies for burst oscillations in this source (Zhang et al. 1998b). The light curve shows no evidence of an X-ray burst or other obvious feature within 1500 seconds before the appearance of the pulsation. Together with its high coherence, this leads us to conclude that the observed pulsation arises from a hot spot spinning with the neutron star. This makes Aql X-1 the millisecond Xray pulsar with the longest orbital period and with the highest time-average accretion rate known at present.

The detection of this fast appearing pulsation in a LMXBs leads once again to the same question: is the pulsation in Aql $\mathrm{X}-1$ usually hidden from the observer, by some scattering or screening medium that very rarely disappears (or strongly reduces its otpical thickness) for $\sim 150$ seconds? Or is the pulsation usually absent, the episode we discovered being the result of an occasional and rare asymmetry on the neutron star surface?

In the following we analyse different possible scenarios:

\subsection{Permanent pulsation}

If a hot spot is normally present on the neutron star surface, the question is what made it observable only for $\sim 150$ seconds. Different authors have argued against or in favor of the hypothesis that in non-pulsating sources the pulsation is washed out by some optically thick scattering media. The main issue is the value of the optical depth $\tau$ needed to attenuate the beamed oscillations. Psaltis \& Chakrabarty (1999) discuss the apparent similarities between values of $\tau$ in pulsating and non-pulsating sources, and discard this hypothesis. Titarchuk et al. (2003) report spectral fits giving other values of $\tau$ (for different sources), and conclude that the data do support this hypothesis. More recently, Gogus et al. (2007) report new, independent spectral fits and conclude that in nonpulsating LMXBs $\tau$ is not large enough to cause the pulsa- tions to disappear (unless the electron temperature is very low, see also Titarchuk et al. 2007).

This issue appears to be difficult to resolve, mainly because of a substantial degeneracy between optical depth and electron temperature in the spectral fits. However, the pulsation episode in Aql X-1 now allows us to provide new insights into this issue. Even though, because of their degeneracy, the absolute values of spectral parameters can not be properly constrained, one would expect them to change when the pulsation appears. In particular, the appearance of the pulsation, if caused by a temporary (total or local) decrease of the optical depth of the scattering media, is expected to be accompanied by a decrease of the comptonization parameter, hence by a softening of the energy spectrum. However, our spectral fits show that a two-component model (disk plus boundary layer) is sufficient to fit the data, without the need for any additional comptonized component. Moreover, neither spectral fitting nor analysis of hardness light curves show any evidence of spectral variability associated with the appearance of the pulsation. Furthermore, the similarities between the spectral properties of Aql X-1 and many other LMXBs would lead one to expect similar episodes of pulsation in most of LMXBs. On the other hand, the very hard spectrum of the observed pulsation (see Fig. 3) suggests that the beamed radiation is strongly comptonized (for a discussion on the expected energy dependence in the case of the screening scenario see Falanga \& Titarchuk 2007).

An alternative could be to assume a non-isotropic screening medium: an occasional and rare hole in the medium or a reflecting cloud could have allowed the radiation from the hot spot to avoid the screening medium, thus becoming visible. Finally, the hot spot itself could have moved becoming temporarily visible, either because of a different absorption through the line of sight or because of a change in the rotational of gravitational lensing geometry. We note that all these hypotheses could in principle be consistent with the observed gradual increase and decrease of the pulsation amplitude, but require very special and fine-tuned geometries.

\subsubsection{Estimate of the magnetic field}

Under the standard dipolar magnetic channeling hypothesis for creating the hot spot, we can give an estimate of the magnetic field. The magnetic field must be strong enough to locally disrupt the disk flow. The pulsation episode happens when the source is in the soft state, close to the peak of the outburst. The $2-10 \mathrm{keV}$ flux is $\sim 8.8 \times 10^{-9} \mathrm{erg}$ $\mathrm{s}^{-1} \mathrm{~cm}^{-2}$ which corresponds, assuming a distance of $5 \mathrm{kpc}$ (Jonker \& Nelemans 2004) and a bolometric correction of 1.4 (Migliari \& Fender 2006), to a bolometric luminosity of $\sim 3.7 \times 10^{37} \mathrm{erg} \mathrm{s}^{-1} \sim 0.15 L_{\mathrm{E}}$. Given the relatively high luminosity, by imposing that the radius at which this happens is larger than the neutron star radius we can derive a stringent lower limit on the magnetic dipole moment (Psaltis \& Chakrabarty 1999) as $\mu \geq 0.47 \times 10^{26} \mathrm{G} \mathrm{cm}^{3}$ which corresponds to a magnetic field of $\sim 10^{8} \mathrm{G}$ at the pole.

This lower limit is consistent with the upper limit of $\sim$ $1.8 \times 10^{9} \mathrm{G}$ obtained by Di Salvo \& Burderi (2003) from the quiescent luminosity and with the estimate of $2 \sim 6 \times 10^{8} \mathrm{G}$ obtained by Campana et al. (1998, assuming a corrected distance of $5 \mathrm{kpc}$ instead of $2.5 \mathrm{kpc}$ ) from the reported onset of the propeller effect. Furthermore, our lower limit rules out the hypothesis of residual accretion onto the neutron star to explain the quiescent X-ray luminosity in Aql X-1, for which an upper limit for the magnetic field of $\sim 4 \times 10^{7} \mathrm{G}$ had been 
derived (hypothesis al of Di Salvo \& Burderi (2003)).

\subsection{Transient pulsation}

The second possibility is that of a temporary asymmetry on the neutron star surface. Let us examine possible causes: a) Magnetic channeling

Accretion rate and magnetic field are the two key physical quantities playing a role in channeling of matter. A transient episode of channeling of matter could arise due to a temporary change in the accretion rate (although the X-ray flux, which is thought to be a good indicator of $\dot{M}$, does not show any variability correlated with the appearance of the pulsation) or a change in the accretion geometry.

An alternative is the occurrence of some variability of the magnetic field. In the "burying" scenario, timescales for the magnetic field to emerge are set by the Ohmic diffusion time and are at least of the order of 100-1000 yr (Cumming et al. 2001), obviously far larger than the few tens of seconds observed in Aql X-1. However a local temporary decrease of the Ohmic diffusion timescale could in principle let the magnetic field emerge. Possible causes could be a temporary local disruption of the screening currents, a starquake, or magnetic reconnections. Perhaps, a temporary local magnetic loop might form, somewhat similar to those observed in the solar corona, where hot coronal plasma "populates" different field lines. The magnetic field in this loop might be locally strong enough to disturb the flow of matter onto the neutron star surface. The resulting hot spot (non necessarily on the magnetic pole) creates the observed pulsation. However multipolar magnetic fields in neutron stars have not been studied in detail, and these phenomena have to our knowledge not been theoretically predicted.

\section{b) Nuclear burning}

The hot spot might be associated to a confined nuclear burning event. After its onset, nuclear burning is expected to spread over the neutron star surface in a very short timescale. Hence one would not expect the resulting pulsation to last more than a few seconds. However the burst oscillations observed in many sources during the decay of X-ray bursts strongly challenge this simplified picture. Moreover, the dis- covery of a very coherent pulsation during a superburst in $4 \mathrm{U}$ 1636-53 (Strohmayer \& Markwardt 2002) showed that longlived, very coherent pulsations might arise due to nuclear burning. However the lack of any other X-ray signature of nulcear burning (such as an X-ray burst) associated with the pulsation in Aql X-1 strongly argues against this hypothesis. c) Surface wave

An asymmetry on the neutron star surface might result from an unstable surface mode, as the ones suggested to explain burst oscillations (see e.g., Piro \& Bildsten 2004; Narayan \& Cooper 2007). Since the amplitudes of surface modes have been predicted to increase with energy (Piro \& Bildsten 2006), the hard energy dependence observed in Aql X-1, more similar to that of burst oscillations than that of accretion-powered pulsations, supports this possibility.

d) Other possibilities

The extreme rarity of this pulsation episode leads us to examine also more exotic physical explanations. Possible origins for a transient hot spot could be a self-luminous magnetic event on the neutron star surface, as magnetic reconnections or annihilations, or a sudden release of potential energy in the deep crust during a starquake.

Finally, we mention the possibility that some of the discussed instabilities could arise due to a collision with an external body (see e.g., Colgate \& Petschek 1981). The fluence of the observed pulsation is $\sim 10^{35} \mathrm{ergs}$, which corresponds to the free fall kinetic energy of a mass of $\sim 15 \mathrm{~km}^{3}$ of iron. However, the observed gradual increase in the pulsation amplitude would be difficult to explain within this scenario. The probability to have such an impact is presumably extremely low. On the other hand, the observed event is very rare.

Whatever is the physical origin of the observed pulsations, their discovery implies the possible occurrance of transient pulsations in any of the apparently non-pulsating LMXBs.

The authors would like to thank A. Gürkan, G. Israel, and L. Stella for very useful comments and stimulating discussions. We also thank L. Bildsten and R. Cooper for pointing out the surface mode possibility.

\section{REFERENCES}

Alpar, M. A., Cheng, A. F., Ruderman, M. A., \& Shaham, J., 1982, Nature, 300, 7128

Altamirano, D., Casella, P., Patruno, A., van der Klis, M., Wijnands, R., 2007, ApJ, submitted

Backer, D. C., Kulkarni, S. R., Heiles, C., Davis, M. M., \& Goss, W. M., 1982, Nature, 300, 615

Brainerd, J., \& Lamb, F. K., 1987, ApJ, 317, L33

Campana, S., Stella, L., Mereghetti S., Colpi, M., Tavani, M., Ricci, D., Dal Fiume, D., \& Belloni, T., 1998, ApJ, 499, L65

Chakrabarty, D., \& Morgan, E. H., 1998, Nature, 394, 346

Chakrabarty, D., 2004, ASP Conference, astro-ph/0408004

Chevalier, C., \& Ilovaisky, S. A., 1991, A\&A, 251, L11

Colgate, S. A., \& Petschek, A. G., 1981, ApJ, 1981, 248, 771

Cumming, A., Zweibel, E., \& Bildsten, L., 2001, ApJ, 557, 958

Di Salvo, T., \& Burderi, L., 2003, A\&A, 2003, 397, 723

Falanga, M., \& Titarchuk, L., 2007, ApJ, 661, 1084

Galloway, D. K., Chakrabarty, D., Morgan, E. H., \& Remillard, R. A., 2002, ApJ, 576, L137

Galloway, D. K., Markwardt, C. B., Morgan, E. H., Chakrabarty, D., \& Strohmayer, T. E., 2005, ApJ, 622, L45

Galloway, D. K., 2006, in AIP Conf. Proc. 840, The Transient Milky Way: A Perspective for MIRAX, ed. J. Braga, F. D'Amico, \& R. E. Rothschild (Melville: AIP), 50

Galloway, D. K., Morgan, E. H., Krauss, M. I., Kaaret, P., \& Chakrabarty, D., ApJ, 654, L73
Gogus, E., Alpar, M. A., \& Gilfanov, M., 2007, ApJ, 659, 580

Illarionov, A. F., \& Sunyaev, R. A., 1975, A\&A, 19, 185

Jonker, P., Nelemans, G., 2004, MNRAS, 354, 355

Kaaret, P., Morgan, E. H., Vanderspek, R., \& Tomsick, J. A., 2006, ApJ, 638, 963

Kylafis, N., \& Klimis, G. S., 1987, ApJ, 323, 678

Leahy, D. A., Darbro, W., Elsner, R. F., Weisskopf, M. C., Kahn, S.,

Sutherland, P. G., Grindlay, J. E, 1983, ApJ, 266, 160

Liu, Q. Z., van Paradijs, J., van den Heuvel, E. P. J., 2007, A\&A, 469, 807

Markwardt, C. B., Swank, J. H., Strohmayer, T. E., in't Zand, J. J. M., \&

Marshall, F. E., 2002, ApJ, 575, L21

Markwardt, C. B., Smith, E., \& Swank, J. H., 2003, IAU Circ. 8080

Markwardt, C. B., \& Swank, J. H., 2003, IAU Circ. 8144

Markwardt, C. B., Krimm, H. A., \& Swank, J. H., 2007, ATel 118

Meszaros, P., Riffert, H., \& Berthiaume, G., 1988, ApJ, 325, 204

Migliari, S., \& Fender, R., 2006, MNRAS, 366, 79

Narayan, R., \& Cooper, R. L., 2007, ApJ, 665, 628

Piro, A. L., \& Bildsten, L., 2004, ApJ, 603, 252

Piro, A. L., \& Bildsten, L., 2006, ApJ, 638, 968

Psaltis, D., \& Chakrabarty, D., 1999, ApJ, 521, 332

Reig, P., Mendez, M., van der Klis, M., \& Ford, E. C., 2000, ApJ, 530, 916 Stella, L., White, N. E., Rosner, R., 1986, ApJ, 308, 669

Strohmayer, T. E., \& Bildsten, L., 2006, in "Compact stellar X-ray sources", eds. W.H.G. Lewin and M. van der Klis, Cambridge University Press, p. $113-156$ 
Strohmayer, T. E., \& Markwardt, C. B., 2002, ApJ, 577, 337

Titarchuk, L., Cui, W., \& Wood, K. S., 2002, ApJ, 576, L49

Titarchuk, L., Kuznetsov, S., \& Shaposhnikov, N., 2007, ApJ, 667, in press

Thorstensen, J., Charles, P., \& Bowyer, S., 1978, ApJ, 220, L131

Welsh, W. F., Robinson, E. L., \& Young P., 2000, AJ, 120, 943

Wijnands, R., \& van der Klis, M., 1998, Nature, 394, 344
Zhang, S. N., Yu, W., \& Zhang, W., 1998a, ApJ, 494, L71

Zhang, W., Jahoda, K., Kelley, R. L., Strohmayer, T. E., Swank, J. H., \& Zhang, S. N., 1998b, ApJ, 495, L9 